\title{
Comparison of the simplex method with several other methods for background-fitting for electron energy-loss spectral quantification of biological materials
}

\author{
Wim De Bruijn $\left({ }^{1}\right)$, Diane Ketelaars $\left({ }^{1}\right)$, Edzard Gelsema $\left({ }^{2}\right)$ and Lianne Sorber $\left({ }^{1}\right)$ \\ (1) AEM-unit, Clinical Pathology I, Erasmus University Rotterdam, Medical Faculty, P.O. Box 1738, \\ 3000 DR Rotterdam, The Netherlands \\ $\left(^{2}\right)$ Dep of Medical Informaticas, Erasmus University Rotterdam, Medical Faculty, P.O. Box 1738, \\ 3000 DR Rotterdam, The Netherlands
}

(Received August 20, 1990; accepted March 26, 1991)

\begin{abstract}
The application of the Simplex optimization procedure allows in functions of the form $I(E)=A^{*} E^{-r}, A$ and $r$ values to be calculated. The use of element-containing Bio-standards with a known externally determined concentration aids in getting reproducible results. Due to the reproducible spectra, fitting-procedures can be compared. The application of a three-dimensional Simplex optimization allows three parameters mutually to be compared. In this way the minimum length of the fitting zone $(\Gamma)$ can be determined in order to find a constant value of $R_{x}$.
\end{abstract}

\section{Introduction.}

Quantification of electron energy-loss (EELS) spectra requires a function of the form $I(E)=$ $A^{*} E^{-r}$ to be fitted over the pre-ionization edge part (PIE) of the spectrum of the element involved, followed by an extrapolation of that function beyond the ionization edge (IE) to acquire the core-loss integral. Three fitting methods are commonly in use:

(1) Egerton's 2-Area method [1].

(2) Bevington's ravine search method, also known as the Steepest descent method $[2,3]$.

(3) Log-Log transformation [1,2]

We will propose the application of Burton's Simplex-optimization method [4,5] as an additional alternative.

To acquire good-quality reproducible spectra, we recently advocated the use of ultrathin sectioned Bio-standard [6-8]. We also suggested calculating in such spectra two types of ratios, called relative concentrations, named $R_{x}^{*}$ and $R_{x}$, of the element involved.

$$
R_{x}=\frac{I_{\mathrm{L}}}{I_{\mathrm{L}}+I_{\mathrm{B}}} \quad R_{x}^{*}=\frac{I_{\mathrm{L}}}{I_{\mathrm{T}}}
$$

$I_{\mathrm{L}}$ is the integral of the part of the edge contributed to by the element involved and $I_{\mathrm{B}}$ is the background integral. $I_{\mathrm{T}}$ is the total integral of the whole spectrum, generally restricted to the 
integral acquired from the first 0-100 eV. The use of multiple spectra from Bio-standards leads to constant $R_{x}$ - and $R_{x}^{*}$-values \pm standard deviation given in percents (=SD\%) [6]. The use of the proposed ratio $R_{x}$ is that the quality of fitting procedures can be judged and/or compared.

Making use of multiple spectra acquired from ultrathin sectioned Bio-standards containing either barium, iron or calcium, the four background-fitting methods mentioned before are compared with respect to the acquired values for: $r, A$ and the relative concentration $R_{x}$.

As a third "unknown" we calculated the minimal fitting-region length $(\Gamma)$ for constant $A, r$ and $R_{x}$ values.

\section{Materials and methods.}

The application of Chelex ${ }^{100}$-ion-exchange beads as standard material loaded with a variety of cations, called Bio-standards, have been described before for X-ray microanalytical purposes [913] and recently for EELS purposes [6-8].

The bead material contains an externally determined (in this case by neutron activation analysis, NAA) element concentration (Bio-Rad/Polaron, Venendaal, NL) and is EPON-embedded with or without tissue. The not by epoxy resin penetrated bead material [14] is sectionable and can be present in the same (untreated) ultrathin section as the tissue containing the unknown concentration of the same element (see [8] for details).

The analytical instrument is a Zeiss 902 (Oberkochen FRG) the instrumental hard and softwareconditions are described somewhere else $[15,16]$.

\section{Results.}

Simplex optimization is a computational strategy to efficiently locate the optimum of a multipleparameter function.

Initially, three pairs of parameter values are chosen, such that the three points in the parameter space $\left(p_{1}, p_{2}\right)$ form an equilateral triangle (the simplex), scaled in both directions by judiciously chosen step sizes. In our application to the EELS spectra the parameter space contains the $A-$ and $r$-values.

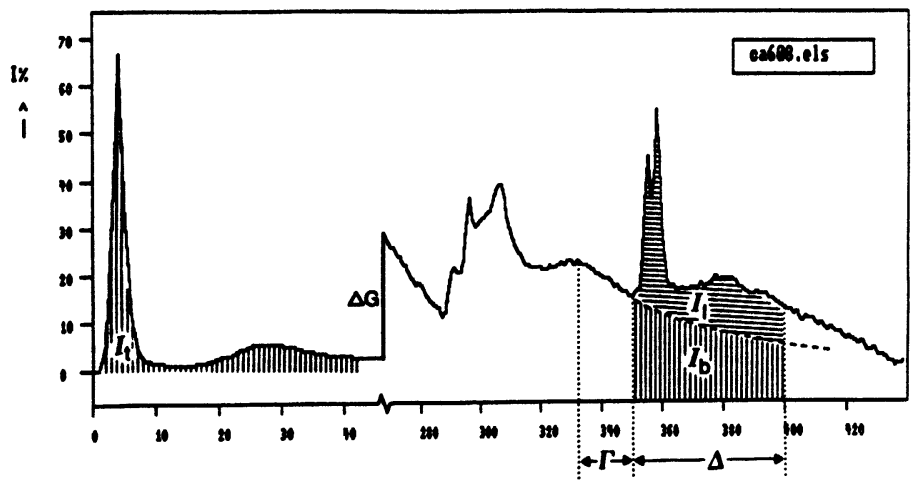

Fig. 1. - Definition of the original $R_{x}^{*}$ value $\left(I_{\mathrm{L}} / I_{\mathrm{T}}\right)$ and the proposed $R_{x}$-value $I_{\mathrm{L}} /\left(I_{\mathrm{L}}+I_{\mathrm{B}}\right)$ (in this case for calcium). 
At these three initially chosen points, a goodness function is calculated, according to $I(E)=$ $A^{*} E^{-\mathrm{r}}$ over all points in the pre-ionization area of the spectrum ( $\Gamma$-range) available. The goodness function $G$, in this application is the sum of squares of deviations of the parametric curve from the spectrum. The merits of this $G$-function are ranked as best (B), next best (N) and worse (W) (See Fig. 2a).

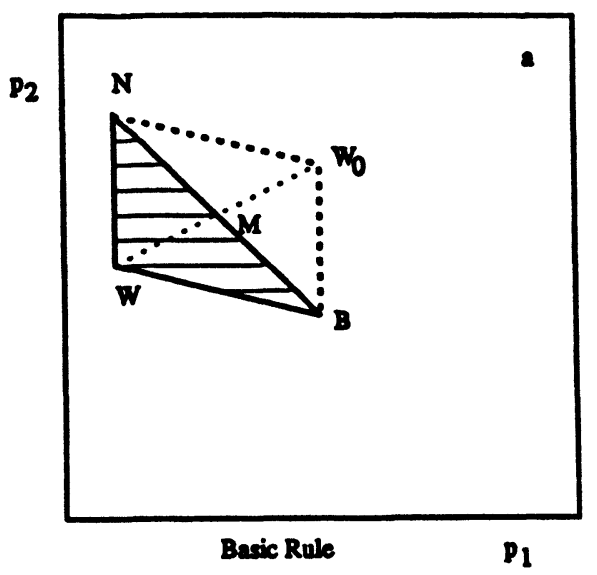

(a)

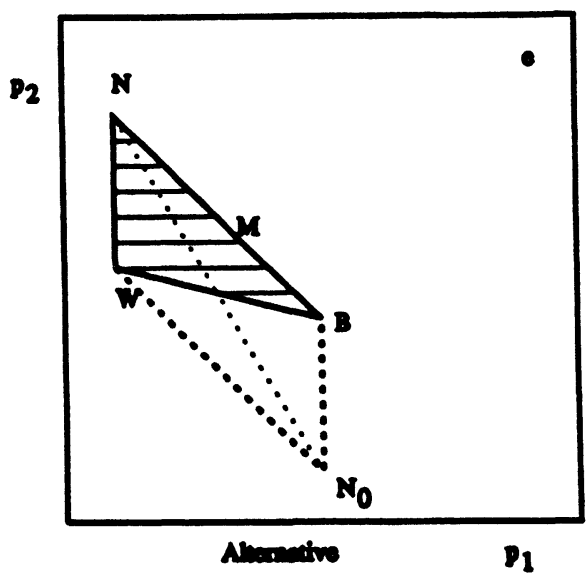

(b)

Fig. 2. - a) Three points in the parameter space $\left(p_{1}, p_{2}\right.$, respectively $A$ and $\left.r\right)$ for which the merits of the Goodness function $G(=$ the sum of squares of deviations of the parametric curve from the spectrum, or the result of the 2-Area method) are ranked as Best (B), Next best (N) and Worse (W). The mid point $M$ is found, which is the reflection centre for $\mathrm{W}\left(\mathrm{WM}=\mathrm{MW}_{0}\right)$. b) An alternative which is applied to the same set of parameters, in which the mid point between $\mathrm{W}$ and $\mathrm{B}$ is found and $\mathrm{N}$ is reflected over that centre to create the alternative point $\mathrm{N}_{\mathrm{o}}$.

The technique then relies on the iterative displacement of the simplex in the parameter space, such that the goodness function $G$ is optimized to a pre-determined accuracy ( Max $_{\text {err }}$, which is the maximum difference accepted by the operator). Details about the basic rule and some alternative rules are illustrated in figures 2-4.

In figures $5 \mathrm{a}$ and $5 \mathrm{~b}$ fitting results are given from an iron-like artificial spectrum, in which the values for $A(=450)$ and $r(=4.5)$ are used to fill a data file. With a preset $\Gamma$-range of $100 \mathrm{eV}$ and a fixed $\Delta$-range of $50 \mathrm{eV}$, the requested values for $A$ and $r$ are subsequently calculated making use of the Simplex optimization method. In figure $5 \mathrm{c}$ a screen dump is given from the simplexes drawn by the program which shows in addition that the known values for $A$ and $r$ were actually calculated. In table I the results are shown of the four methods compared for the same test-spectrum. The Steepest descent results were acquired here by introduction of the 2 -area values as starting points for this method.

Subsequently, a true barium spectrum from a Ba-Bio-standard (shown in Figs. 6a and 6b) was treated similarly, with the simplexes shown in figure $6 \mathrm{c}$.

Further we investigated for all four methods the minumum $\Gamma$-range necessary to get constant $A$, $r$ and $R_{x}$ values. In figure 7 we copied such a situation for the Simplex-method from the original paper [4] in which a three parameter space is present and the original simplex is replaced by an equilateral in which $W$ is reflected over point $P$ to give a new pyramid $R N_{1} N_{2} B$. 


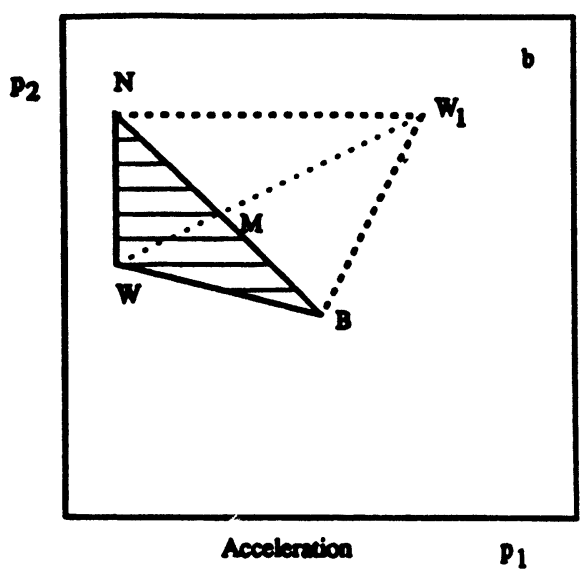

Fig. 3. - Shows an acceleration step in which after the merits $\mathrm{W}_{0}\left(\mathrm{WM}=\mathrm{MW}_{0}\right)$ are established also $\mathrm{W}_{1}$ $\left(\mathrm{MW}_{1}=2^{*} \mathrm{WM}\right)$ is calculated and compared to the result acquired for $\mathrm{W}_{0}$.

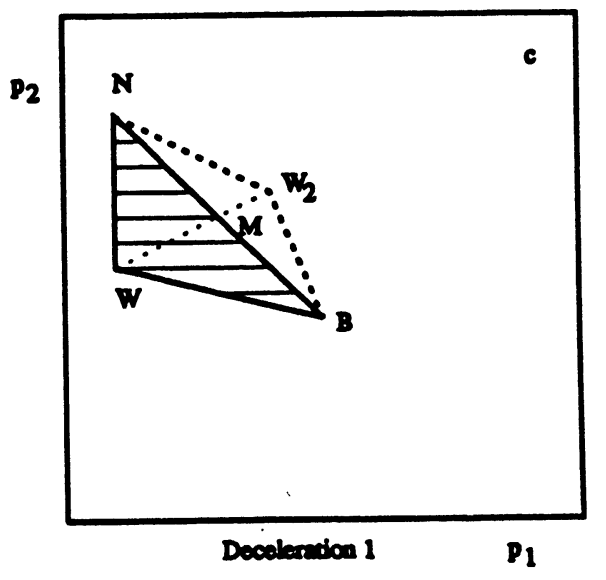

(a)

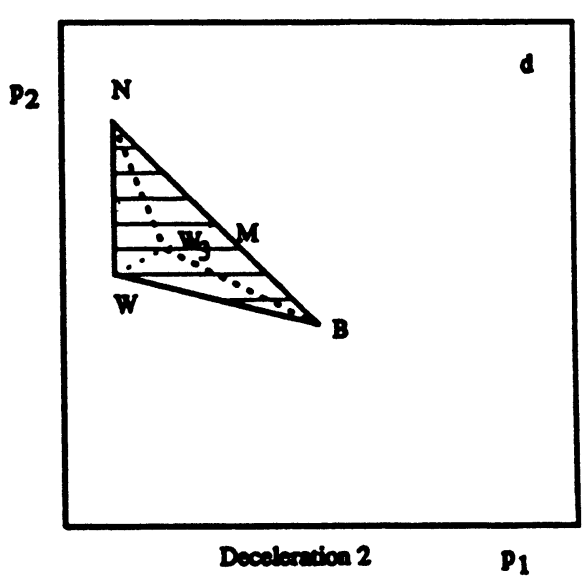

(b)

Fig. 4. - Show two deceleration steps in the vicinity of the final result, in which in addition to the merits established for $W_{0}$, those are calculated for $W_{2}\left(W M=2^{*} W_{2}\right)$ and $W_{3}\left(W M=2^{*} W W_{3}\right)$.

In figures $8 \mathrm{a}$ to $8 \mathrm{c}$ the $A, r$ and $R_{\mathrm{Fe}}$-results are depicted for the artificial spectrum shown in figure 5 with increasing lengths of the $\Gamma$-range and a fixed $\Delta$-range of $50 \mathrm{eV}$. In this picture also the results of Egerton's two-area method are depicted, treated in a similar way. Using the Simplexmethod after 15-20 eV all three values are constant.

In figure 9 the $R_{\mathrm{Ba}}$-value is shown for the $\mathrm{Ba}$-spectrum from figure $6 \mathrm{a}$, for the same conditions and in which the Simplex is compared to the 2-Area method. In table II the mean values are given for four methods, compared from 28 iron-spectra, all recorded in one ultrathin section.

A situation in which this approach could be fruitful, is when two IE's are (too) close together. this is the case with the carbon- and calcium-edge. In figures $10 \mathrm{a}$ to $10 \mathrm{e}$ a similar set of results 

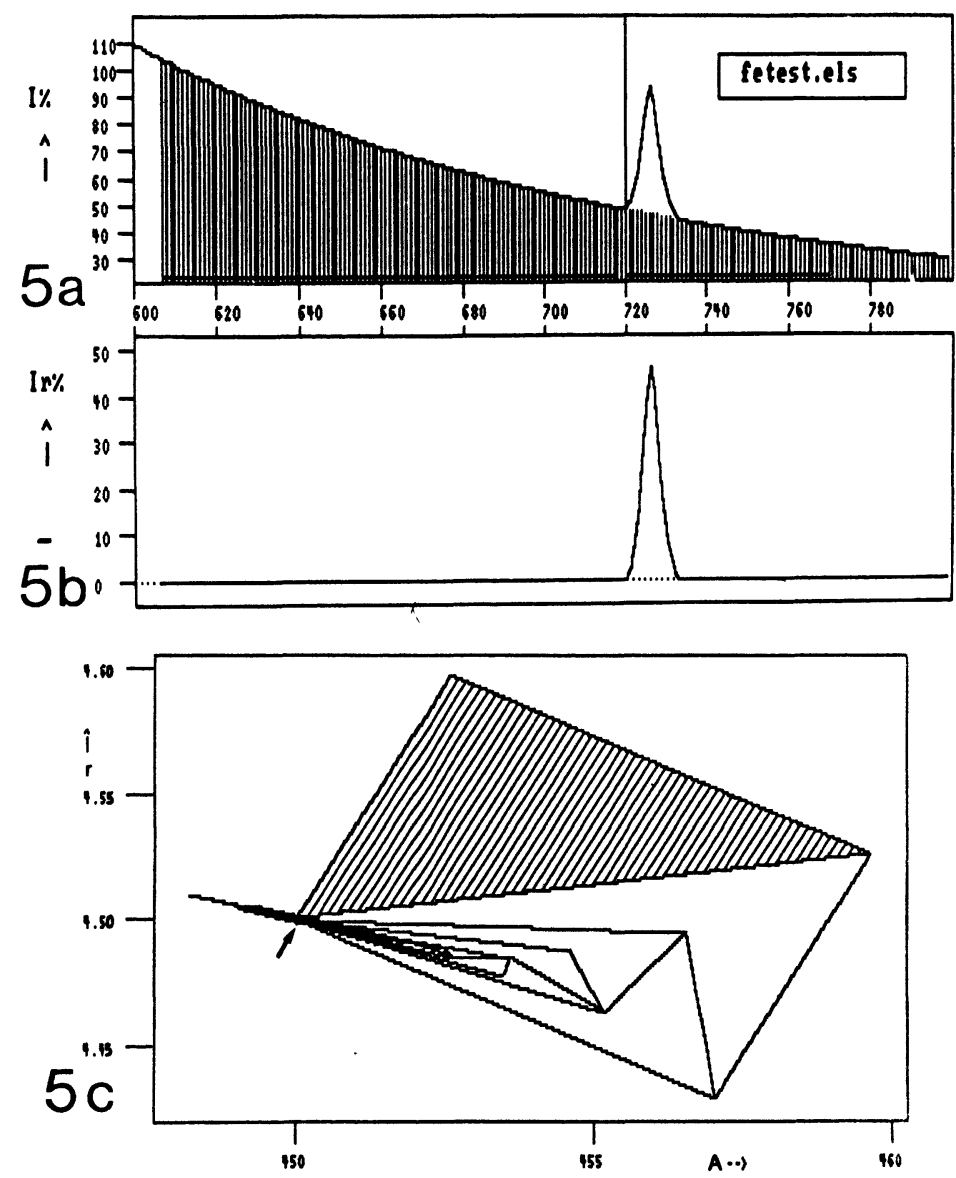

Fig. 5. - Shows an artificial iron-like spectrum which was acquired by filling a data file with $A=450$, and $r=-4.5$. 5a the complete spectrum, $5 \mathrm{~b}$ the subtracted spectrum, $5 \mathrm{c}$ screen dump from the simplexes drawn by the program while finding the correct $A$ and $r$ values (arrow).

Table I. - Mean $A, r, R_{x}$-values $\pm S D \%$ of a test-spectrum $(A=450, r=4.5)$.

\begin{tabular}{|l|c|c|c|}
\hline method & $A$ & $r$ & $R_{x}$ \\
\hline 2-Area & 449.0 & 4.50 & 0.204 \\
Log - Log & 450.0 & 4.50 & 0.202 \\
Steepest Descent & 447.6 & 4.51 & 0.204 \\
Simplex & 450.0 & 4.50 & 0.202 \\
\hline
\end{tabular}

is shown of a calcium Bio-standard. The $\Gamma$-range available is just too short to ascertain that the $R_{\mathrm{Ca}}$ remains constant. Beyond $23 \mathrm{eV}$ for the $\Gamma$-range the values deteriorate again. Table III gives the mean values from 28 calcium-spectra, recorded in one ultrahin section, of the four methods $(\Gamma=23 \mathrm{eV}, \Delta=50 \mathrm{eV})$. 

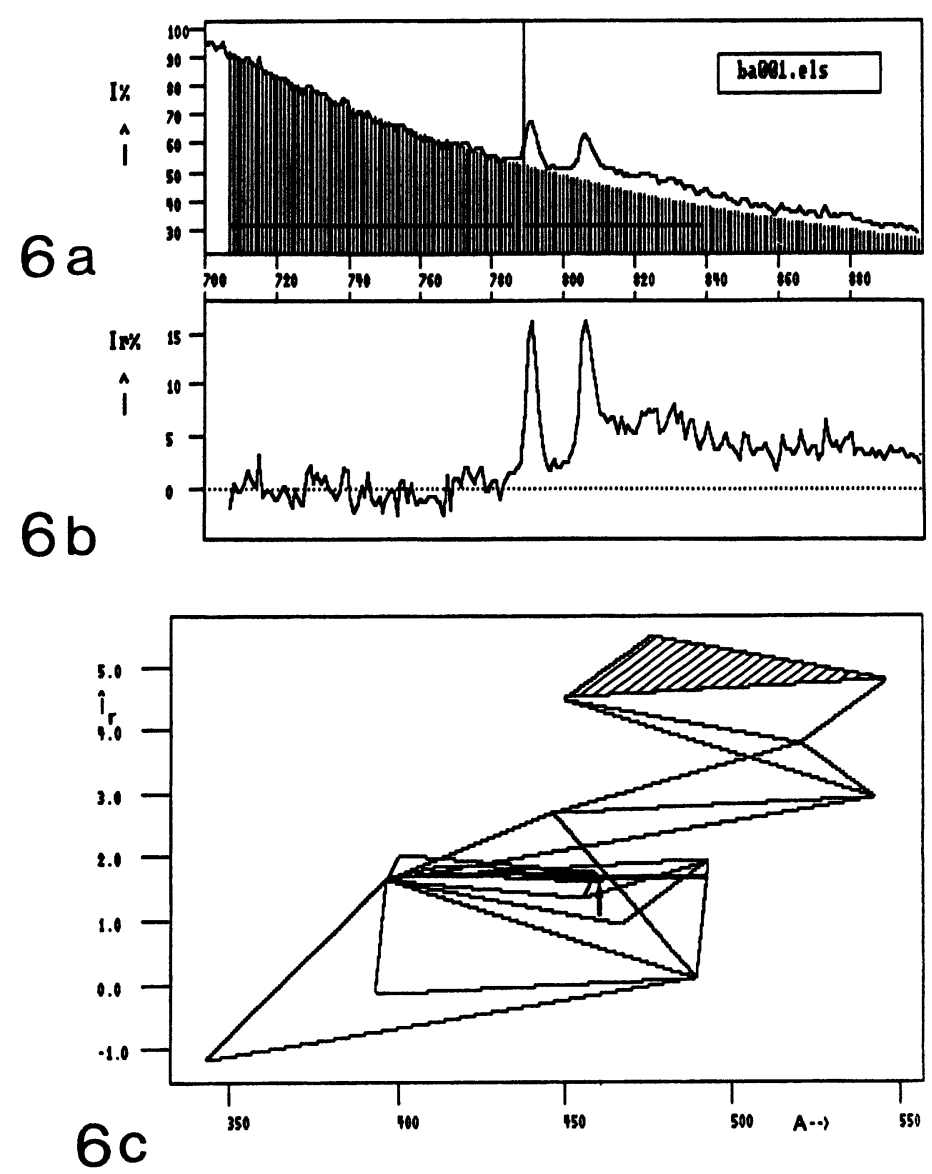

Fig. 6. - A similar result is shown for a Ba-Bio-standard. 6a, the complete spectrum, $6 \mathrm{~b}$, the subtracted spectrum, $6 \mathrm{c}$, the simplexes drawn to find the $A$ and $r$ values (arrow) for a Maxerr $=10^{-6}$.

In conclusion, it has been shown that with the Simplex method, the three values $A, r$ and $R_{x}$ acquire sooner a constant level than for example the 2-Area method when, at a fixed value of the integration region $(\Delta=50 \mathrm{eV})$, the width of the fitting region $(\Gamma)$ is continuously increased. The proposed Simplex method fits a curve through all data points available in the original function. Like the Steepest descent method, its accuracy can be adapted, be it that the speed of the fitting procedure is considerably reduced. The Log-Log method, which fits a straight line through the logarithm of the function gives results comparable to those of the Simplex method although with the calcium-spectra there is a small difference, slightly favouring the use of the Simplex method. However the Log-Log method is faster than the Simplex method.

This 3-dimensional Simplex allows a reduction of the width of the fitting region to $30-35 \mathrm{eV}$ for the elements tested (Fe, $\mathrm{Ba}$ ) for constant $R_{x}, r$ and $A$-values. However the nominal $\Gamma$-range of $23 \mathrm{eV}$ available is just inadequate to ascertain that constant $r, A$ and $R_{x}$-values are acquired for calcium. 


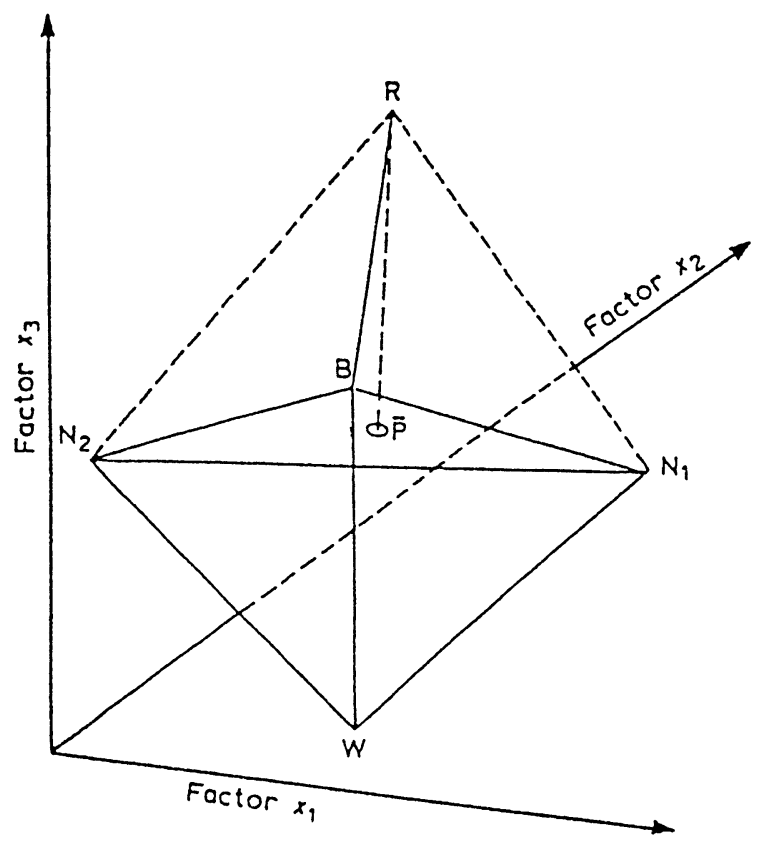

Fig. 7. - A three-dimensional Simplex parameter space. $X_{1}=A, X_{2}=r, X_{3}=$ length of the $\Gamma$ range.

\section{Discussion.}

The application of Bio-standards can be divided in three main fields: (a) To acquire spectra from various elements in ultrathin sections; (b) To compare instrumental and, as shown here, computerprogram conditions; (c) To calculate elemental concentrations by $R_{x}^{*}$-comparison between "standard" and "unknown".

Since their introduction in 1981 for electron probe X-ray microanalyses (EPMA) [9] several aspects have been elucidated also for other analytical techniques $[12,14,17]$.

By the introduction of their use for EELS-analyses [18] attention was predominantly focused upon the first aspect (a). Recently, the use for the aspects (b and c) have been demonstrated $[7,8,19]$.

The initial historical Bio-standard application for EPMA was based upon the comparison between $R_{x}$-values (Peak- Background/Background) acquired in the "known" standard and that from the "unknown". There it was noticed that $R_{x}$-values from the Bio-standards were constant within a certain range $[11,20]$.

This induced the idea also to look for the constancy in EELS spectra from Bio-standards for the $R_{x}$-value as defined in figure 1 (or alternatively to the ratio $I_{\mathrm{L}} / I_{\mathrm{B}}$ ) although it was realized that for quantitative spectral analysis already a relative concentration was defined $\left(R_{x}^{*}\right)$ before [21].

The aplication of the two-dimensional Simplex optimization method allowed us to find $A$ and $r$ irrespective of the accuracy that was preset. So either both Two-area methods (geometric or arithmetic mean) or other fitting methods (minimizing the sum of squares) could be introduced and the results and values of $A$ and $r$ compared.

From the comparison of the four methods we can conclude that comparable results were ac- 


\section{Simplex vs 2-Area} fetest

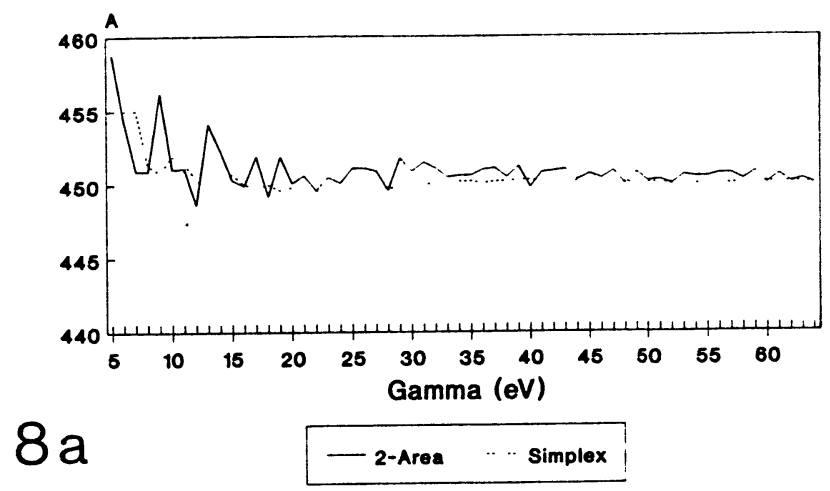

\section{Simplex vs 2-Area fetest}

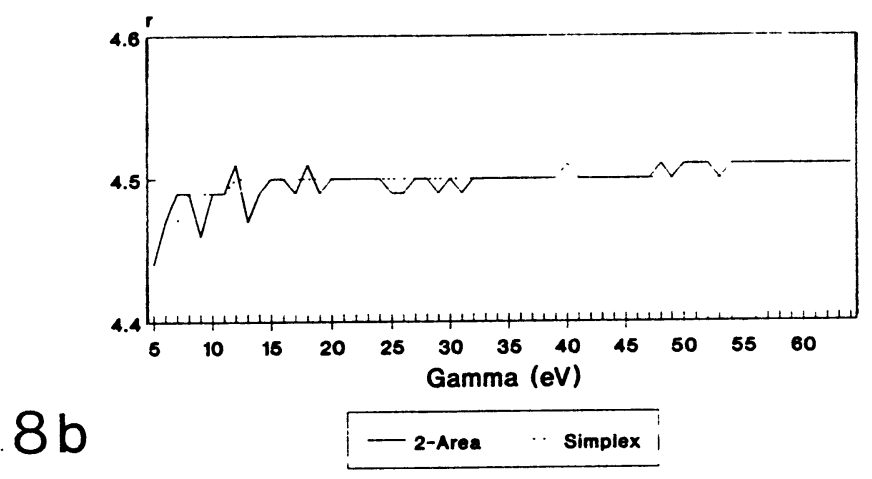

\section{Simplex vs 2-Area}

Fetest

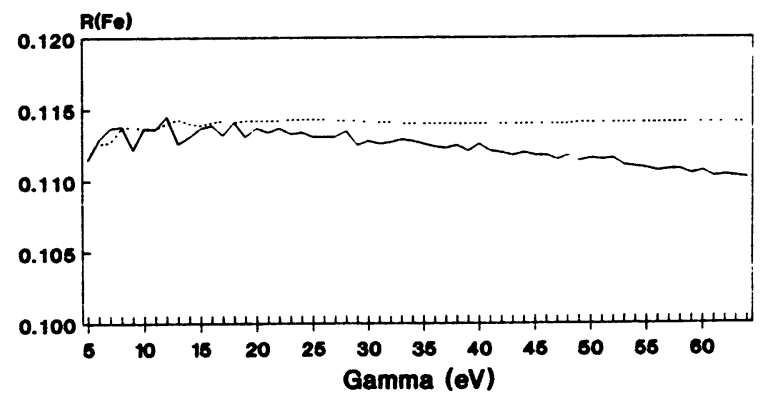

$8 c$

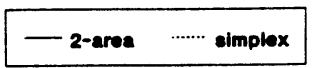

Fig. 8. - The $A$, the $r$ and the $R_{\mathrm{Fe}}$ values are shown for the test-spectrum shown in figure 5a. The results of the Simplex-method are compared to the application of the 2-area method also with a $\Delta=50 \mathrm{eV}$ and an increasing $\Gamma$-range. 


\section{Ba001.els}

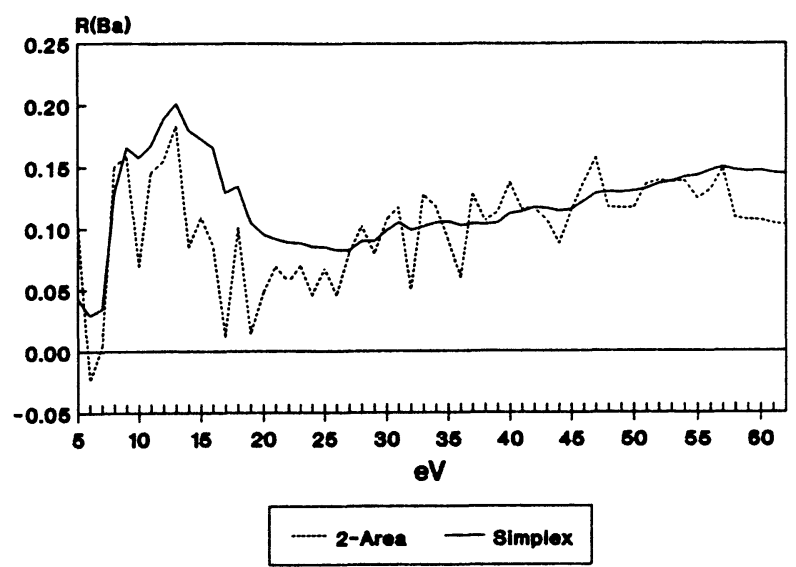

Fig. 9. - The $R_{\mathrm{Ba}}$ values for the spectrum shown in figure 6, compared to the 2-area method results.

Table II. - Mean A, $r, R_{x}$-values $\pm S D \%$ of 28 Fe-Bio-standard-spectra.

\begin{tabular}{|l|c|c|c|}
\hline method & $A$ & $r$ & $R_{x}$ \\
\hline 2-Area & $363.1 \pm 27 \%$ & $4.37 \pm 8 \%$ & $0.222 \pm 19 \%$ \\
Log - Log & $362.1 \pm 27 \%$ & $4.40 \pm 10 \%$ & $0.220 \pm 20 \%$ \\
Steepest Descent & $336.5 \pm 40 \%$ & $4.12 \pm 28 \%$ & $0.201 \pm 36 \%$ \\
Simplex & $366.3 \pm 29 \%$ & $4.39 \pm 11 \%$ & $0.218 \pm 22 \%$ \\
\hline
\end{tabular}

quired for iron by all four methods applied, because iron has a long $\Gamma$-range with a clear inverse power law function. Only for the Steepest descent method the standard deviation is larger than for the other three methods.

For calcium the 2-Area method and the Steepest descent method can not fit a proper background. The Log-Log method and the Simplex method have better results, but still with high standard deviations as compared to the SD\% acquired for the iron-spectra.

Application of a three dimensional Simplex method (see Fig. 7) allowed us to judge the minimal number of channels in the $\Gamma$-range that was needed to get constant values for $A$, and $r$ (for $\mathrm{Ca}$ and Fe-Bio-standards see [7,8]). As shown here also constant $R_{x}$ values were acquired from: testspectra (Figs. 8a-c), real Ba, Fe and Ca spectra. 


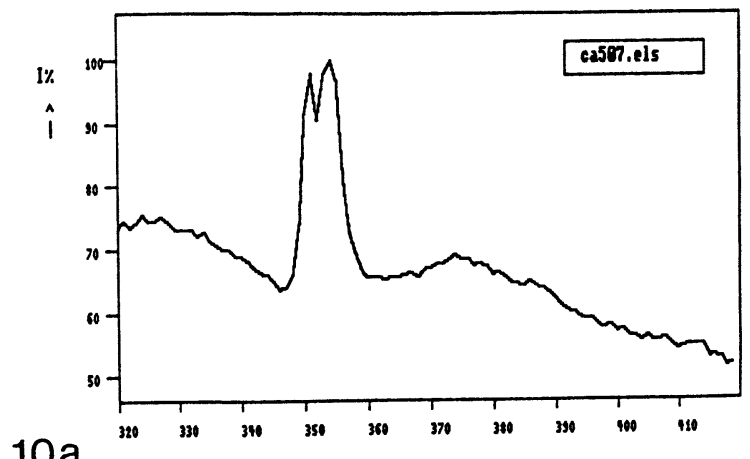

$10 a$

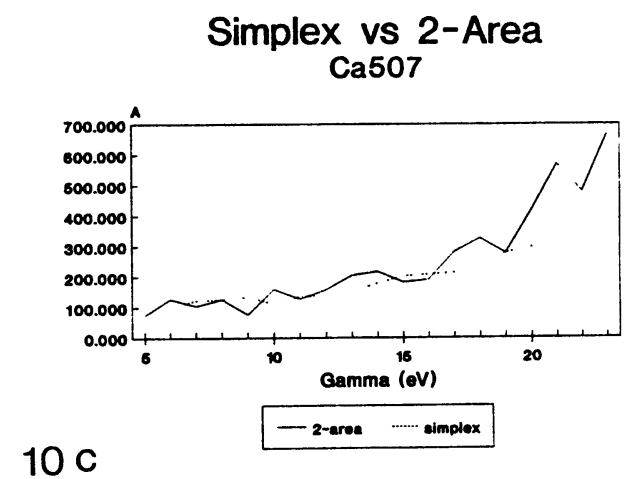

$10 \mathrm{c}$

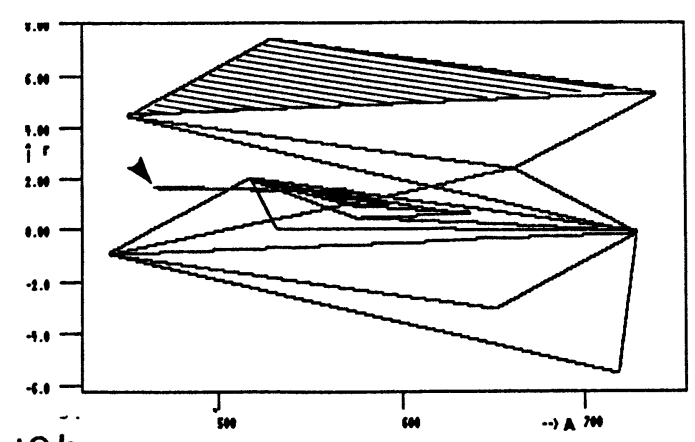

$10 b$

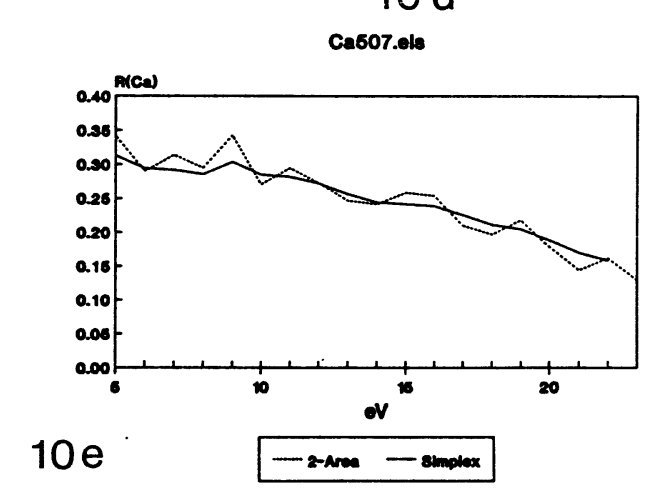

Simplex vs 2-Area Ca507

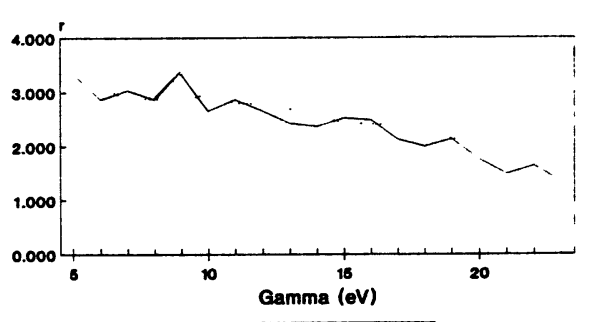

$10 d$

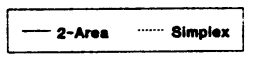




\section{References}

[1] EgERTON R.F., Energy Loss-Spectroscopy in the electron microscope (Plenum Press) 1986, p. 257.

[2] Bevington P.R., Data reduction and error analysis for the physical sciences (Mc Graw-Hill book company) 1969, p. 246.

[3] CollieX C., MANOUBI T. and KRIVANEK O.L., J. Electron Microsc. 35/4 (1986) 307-313.

[4] BURTON K.W.C. and NICKLESS G., Chemometrics intelligent lab. syst. 1 (1987) 135-149.

[5] MORGAN E., BURTON K. and NiCKLESS G., Chemometrics Intelligent Lab. Syst. 7 (1990) 209-222.

[6] De BruiJn W.C. and SORBER C.W.J., Proc. XII Ih Intern. Congr. Electron Microsc. Seattle 2 (San Francisco Press Inc.) 1990, 60.

[7] Sorber C.W.J., KeTELAARs G.A.M., GElSEMA E.F., Jongkind J.F. and De BruiJN W.C., Proc. $2^{\text {nd }}$ ESI-workshop Dortmund 1990, J. Microsc. 162 (1991) 23.

[8] Sorber C.W.J., KetelaARs G.A.M., Gelsema E.F., Jongkind J.F. and De BruiJN W.C., Proc. $2^{\text {nd }}$ ESI-workshop Dortmund 1990, J. Microsc. 162 (1991) 43.

[9] De BRuiJn W.C., Scanning Electron Microsc. II (SEM Inc., A.M.F. O'Hare) 1981, p.357.

[10] De BRUIJN W.C., Scanning Electron Microsc. II (SEM Inc., A.M.F. O’Hare) 1985 p.697.

[11] De BRUIJ W.C. and CLETON-SOETEMAN M.I., Scanning Electron Microsc. II (SEM Inc., A.M.F. O'Hare) 1985 p.715.

[12] De Bruijn W.C., Koerten H.K., Cleton-Soeteman M.I. and BloK-Van Hoek C.J.G., Scanning Microsc. 1/4 (Scanning Microscopy international, A.M.F. O'Hare) 1987, p.1651.

[13] De BRUIJN W.C. and VAN MIERT M.P.C., Scanning Microsc. 2 (SEM Inc., A.M.F. O'Hare) 1988 p.319.

[14] Verbueken A.H., VAN GRIEKEN E., Poulas G.J. and De Bruijn W.C., Anal. Chem. 56 (1984) 1362.

[15] SORBER C.W.J., de JONG A.A.W., DEN BREEJEN N.J. and De BRUIJN W.C., Ultramicrosc. 32 (1990) 55.

[16] Sorber C.W.J., VAN DORT J.B., Ringeling P.C., Cleton-SOETEMaN M.I. and De BRUiJn W.C., Ultramicrosc. 32 (1990) 69.

[17] Cleton M.I., Roelofs J.M., Block-Van Hoek C?J?G? and De BruiJn W.C., Scanning Electron Microsc. 1986/III (SEM Inc., A.M.F. O’Hare) 1986 p.999.

[18] SORBER C.W.J., VAN DORT J.B. and De BRUIJN W.C., Proc. EMAS EUropean Microanalysis Soc. (1989) 192.

[19] De Bruijn W.C., KetelaArs D., Vermeij M., CaO L.C., Bouvé E. and Schröder F.H., Urological Research 18/1 (1990) 62.

[20] ROOMANS G.M. and SHELBURNE J.D., Scanning Electron Microsc. II (SEM Inc. A.M.F. O'Hare) 1980) p.309.

[21] EGERTON R.F., Energy Loss-Spectroscopy electron microsc.(Plenum Press) 1986 p.78. 